\title{
An Extension of Taylor's $\varphi$-Circle Method and Some Stability Charts for Submerged Slopes
}

\author{
Ping Li $\mathbb{D}^{1,2}$ Luanhua Dong, ${ }^{1,2}$ Xiaowen Gao, ${ }^{3}$ Tonglu Li, ${ }^{1,2}$ and Xiaokun $\mathrm{Hou}^{2,4}$ \\ ${ }^{1}$ School of Geological Engineering and Geomatics, Chang'an University, Xi'an 710054, Shaanxi, China \\ ${ }^{2}$ Water Cycle and Geological Environment Observation and Research Station for the Chinese Loess Plateau, \\ Ministry of Education, Gansu 745399, China \\ ${ }^{3}$ Electronic Comprehensive Investigation Surveying Institute of the Ministry of Information Industry Xi'an, Xi'an 710054, \\ Shaanxi, China \\ ${ }^{4}$ Key Laboratory of Shale Gas and Geoengineering, Institute of Geology and Geophysics, Innovation Academy for Earth Science, \\ CAS, Beijing 100029, China
}

Correspondence should be addressed to Ping Li; dcdgx07@chd.edu.cn

Received 20 September 2019; Accepted 15 February 2020; Published 28 August 2020

Academic Editor: Chao Wu

Copyright ( 2020 Ping Li et al. This is an open access article distributed under the Creative Commons Attribution License, which permits unrestricted use, distribution, and reproduction in any medium, provided the original work is properly cited.

\begin{abstract}
Taylor's $\varphi$-circle method is a classical method for slope stability calculation, which has analytical solutions. Taylor derived equations in two cases separately, namely, (i) the outlet of the critical failure surface is at the slope toe and (ii) the outlet of the failure surfaces is not at the slope toe. The method is only appropriate for two conditions (without underground water table in slopes or totally submerged slopes). In this study, a general equation that unifies the equations of the two cases is proposed and partially submerged condition is introduced. The critical failure surfaces corresponding to the minimum factor of safety are determined using the computer program proposed by the authors. The general expression of the safety factor of slopes under the following four conditions is derived, namely, (i) partly submerged, (ii) completely submerged, (iii) water sudden drawdown, and (iv) water slow drawdown. The corresponding charts for practical use are available.
\end{abstract}

\section{Introduction}

The $\varphi$-circle method proposed by Taylor [1] is one of the classical methods of slope stability calculation, which is suitable for homogeneous soil slopes (i.e., without underground water table in slopes or totally submerged slopes). It is assumed that the potential failure surface is a circular arc and the resultant force of the friction and normal stress on the entire failure surface is tangent to the $\varphi$-circle. The $\varphi$-circle is a circle which is concentric with the failure surface. The radius of the $\varphi$-circle is the product of the radius of failure surface $\operatorname{arc} R$ and $\sin \varphi$. Thus, this method is termed the $\varphi$-circle method. This $\varphi$-circle method regards the whole mass above the failure surface as a research object and has strict mathematical derivation and analytical solution. In addition, this method follows both the force and moment equilibriums. In the $\varphi$-circle method, the dimensionless term $N$, which is related to the cohesion, unit weight, the factor of safety, and the slope height, is termed the "stability number." The expression for stability number proposed by Taylor [1] is simple and intuitive. The results are easily expressed in the chart. The method is generally used in the homogeneous and isotropic simple slope analysis and has been extended to some special cases, such as spatial variable strength parameters $[2,3]$, linearly increasing cohesion with depth [4-7], quasistatic seismic effect [8-10], and slope couple foundation stability research [11]. Taylor's charts have been re-examined and developed using finite-element limit analysis and elastic-plastic finite-element method [12-15]. The advantage of the numerical methods is that they have no assumptions about the shape and the location of the failure surface. It has been found that Taylor's original stability number is "reasonably accurate" when the failure surface is circular, but differs more than $10 \%$ when the 
failure surface is not circular. Some numerical solutions have been developed to extend the slope stability chart in three dimensional slopes [16-18] and heterogeneous slopes [19].

Cojean and Fleurisson [20] and Cojean and Cai [21] have well documented the effect of water level fluctuation on the factor of safety of slopes. They analyzed the factor of safety under the conditions of static water level and water sudden drawdown in graphical form. Li et al. [22] presented a landslide case on reservoir bank which shows that the factor of safety decreases firstly and then increases with the rise of water level, which agrees with the results of Cojean and Fleurisson [20]. The analytical solution for water drawdown slopes still is not available.

In this study, the original Taylor's $\varphi$-circle method is modified, which unifies the original equations in two cases into one general equation. Then, analytical solutions for the factor of safety of slopes subjected to the water drawdown are derived. Four cases are considered, namely, (i) partially submerged slopes, (ii) completely submerged slopes, (iii) water sudden drawdown, and (iv) water slow drawdown. Searching for the critical failure surface according to the minimum factor of safety was carried out using the computer program that the authors proposed. The charts of the factor of safety versus slope inclinations and water levels are provided to illustrate the effect of water level on the slope stability.

\section{Modified Taylor's $\varphi$-Circle Method}

Figure 1 shows a typical slope model. In this model, $\mathrm{AB}_{1} \mathrm{C}_{1}$ represents the potential sliding mass; $H_{1}$ is the slope height; $\beta$ is the slope angle; $2 \alpha_{1}$ is the central angle of the failure surface arc $A B_{1} ; \lambda_{1}$ is the inclination angle of the chord $A B_{1}$; and $\mathrm{nH}_{1}$ is the distance between the slope toe and the outlet of potential failure surface. When $n=0$, the outlet of the potential failure surface is just at the slope toe. The radius $r$ of the $\varphi$-circle equals $R \sin \varphi . \varphi$ is the internal friction of the failure surface.

From the geometry of Figure 1, the radius $R$ of the failure surface arc is

$$
R=\frac{H_{1}}{2} \csc \alpha_{1} \lambda_{1}
$$

According to Taylor's derivation, the gravity of the potential sliding mass $\mathrm{AB}_{1} C_{1}$ is

$$
\begin{aligned}
W_{1}= & \frac{\gamma_{1} H_{1}^{2}}{4}\left[\alpha_{1} \csc ^{2} \lambda_{1} \csc ^{2} \alpha_{1}-\csc ^{2} \lambda_{1} \cot \alpha_{1}\right. \\
& \left.+2\left(\cot \lambda_{1}-\cot \beta\right)\right]-\gamma_{1} n H_{1}^{2},
\end{aligned}
$$

where $\gamma_{1}$ is the unit weight of the soil above the water level. Let

$$
f\left(\lambda_{1}, \alpha_{1}\right)=\left(\frac{1}{2}\right) \csc ^{2} \lambda_{1}\left(\alpha_{1} \csc ^{2} \alpha_{1}-\cot \alpha_{1}\right)+\cot \lambda_{1}-\cot \beta .
$$

Equation (2) is simplified as

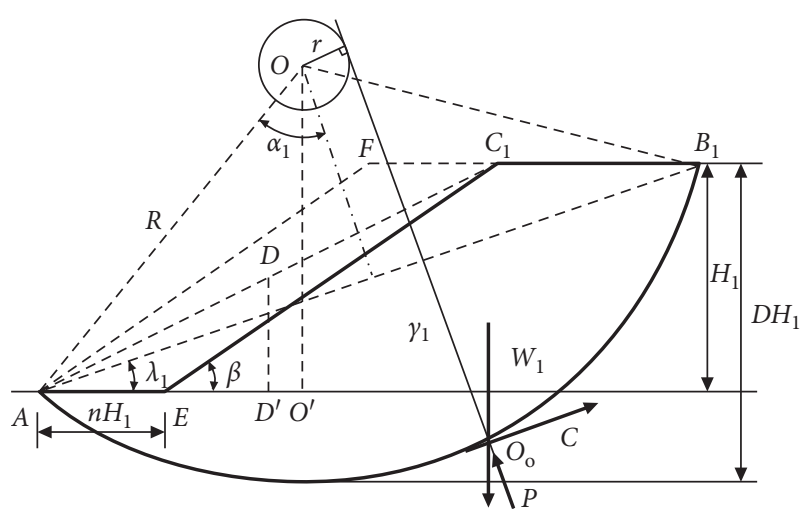

Figure 1: A simple slope model (adapted from Taylor [1]).

$$
W_{1}=\left(\frac{\gamma_{1} H_{1}^{2}}{2}\right) f\left(\lambda_{1}, \alpha_{1}\right)-\gamma_{1} n H_{1}^{2} .
$$

The gravity moment of potential sliding mass $\mathrm{AB}_{1} C_{1}$ is

$$
\begin{aligned}
W_{1} d_{1}= & \left(\frac{\gamma_{1} H_{1}^{3}}{12}\right)\left[1-2 \cot ^{2} \beta+3 \cot \lambda_{1} \cot \beta-3 \cot \alpha_{1} \cot \beta\right. \\
& \left.+3 \cot \lambda_{1} \cot \alpha_{1}\right]-\left(\frac{\gamma_{1} H_{1}^{3}}{4}\right)
\end{aligned}
$$$$
\cdot\left(2 n^{2}-2 n \sin \phi \csc \lambda_{1} \csc \alpha_{1}\right) \text {, }
$$

where $d_{1}$ is the arm of gravity to the center of the $\varphi$-circle.

Taylor has derived the equations in two cases, namely, (i) the outlet of the failure surface is at the slope toe (i.e., $n=0$ ) and (ii) the outlet of the failure surfaces is not at the slope toe (i.e., $n>0$ ). When $n>0$, the failure arc has the minimum factor of safety when the right vertical tangent of the $\varphi$-circle passes through the midpoint of the slope $C_{1} E$. When the center of the failure arc is limited to the vertical line on the left of the midpoint of $C_{1} E, n$ can be calculated with the condition. It will reduce one degree of freedom in searching the failure surface, significantly alleviating the computation work. However, with the development of the computation ability of the computer, it is not difficult to search the failure surface with total degrees of freedom nowadays. In this study, a general equation of moment is derived here which unifies the equations in the two cases, namely, $n=0$ and $n>0$. Thus, $n$ is one degree of freedom for searching the failure surface, which is convenient for programming.

In Figure $1, \mathrm{AEC}_{1} F$ is a parallelogram, whose center of gravity lies in the midpoint of the line $\mathrm{AC}_{1}$. The arm of $\mathrm{AEC}_{1} F$ to the center of the $\varphi$-circle can be generally expressed as

$$
D^{\prime} O^{\prime}=\left(\frac{1}{2}\right)\left(H_{1} \cot \beta+n H_{1}\right)-R \sin \left(\alpha_{1}-\lambda_{1}\right)
$$

The gravity of parallelogram $\mathrm{AEC}_{1} \mathrm{~F}$ is $\gamma_{1} n H_{1}^{2}$, and substituting equation (1) into equation (6), the gravity moment is 


$$
\frac{\gamma_{1} n H_{1}^{3}}{2}\left(\cot \beta+n-\cot \lambda_{1}+\cot \alpha_{1}\right)
$$

Replacing the last term of equation (5) with the equation (7), the general expression for the gravity moment of the sliding mass $\mathrm{AB}_{1} C_{1}$ is

$$
\begin{aligned}
W_{1} d_{1}= & \left(\frac{\gamma_{1} H_{1}^{3}}{12}\right)\left[1-2 \cot ^{2} \beta+3 \cot \lambda_{1} \cot \beta-3 \cot \alpha_{1} \cot \beta\right. \\
& \left.+3 \cot \lambda_{1} \cot \alpha_{1}\right]-\left(\frac{\gamma_{1} n H_{1}^{3}}{2}\right) \\
& \cdot\left(\cot \beta+n-\cot \lambda_{1}+\cot \alpha_{1}\right) .
\end{aligned}
$$

$$
\begin{aligned}
& \text { Let } \\
& \begin{aligned}
\psi\left(\lambda_{1}, \alpha_{1}\right)= & \cot \beta+\cot \alpha_{1}-\cot \lambda_{1}, \\
g\left(\lambda_{1}, \alpha_{1}\right)= & \frac{1}{3}\left(1-2 \cot ^{2} \beta\right)+\cot \beta\left(\cot \lambda_{1}-\cot \alpha_{1}\right) \\
& +\cot \lambda_{1} \cot \alpha_{1} .
\end{aligned}
\end{aligned}
$$

Then, equation (8) can be simplified as

$$
W_{1} d_{1}=\left(\frac{\gamma_{1} H_{1}^{3}}{4}\right) g\left(\lambda_{1}, \alpha_{1}\right)-\left(\frac{\gamma_{1} n H_{1}^{3}}{2}\right)\left[\psi\left(\lambda_{1}, \alpha_{1}\right)+n\right] \text {. }
$$

With modified equation (11), the stability number can be derived in exactly the same way as Taylor's derivation.

\section{The Factor of Safety in Water Drawdown Slopes}

The submerged slope is shown in Figure 2. When water level declines from a height of $\mathrm{H}_{2}$ to $\mathrm{H}_{3}$ as shown in Figure 3, the corresponding depth of water increases from $D_{2}$ to $D_{3}$. Supposing there is no water recharge at the inner side of the slope, the pore water pressure on the failure surface can be expressed by the following equation:

$$
\begin{aligned}
& u=r_{u} \gamma_{1}\left(D-D_{2}\right), \\
& r_{u} \subset\left[0, \frac{\gamma_{w}}{\gamma_{1}}\right],
\end{aligned}
$$

where $D$ is the depth at the failure surface between the water level before drawdown $\left(\mathrm{H}_{2}\right)$ and water level after drawdown $\left(H_{3}\right), u$ is the pore water pressure at the depth of $D, r_{u}$ is the pore water pressure coefficient, and $\gamma_{w}$ is the unit weight of water.

Two extreme situations are considered. One is that the sliding mass is completely impermeable, so the soil between the water level before drawdown $\left(H_{2}\right)$ and after drawdown $\left(H_{3}\right)$ should be saturated. The pore water pressure on the failure surface can be calculated by the difference of $\mathrm{H}_{2}$ and $H_{3}$. The other case is that the sliding mass is completely permeable, and the pore water pressure on the failure surface is approximately zero. The general case is between the two extreme conditions, so the average unit weight of the soil between the water levels before and after drawdown is estimated by interpolating between the saturated unit weight and the unsaturated unit weight as

$$
\gamma_{a}=\gamma+r_{u} \frac{\gamma}{\gamma_{w}}\left(\frac{e}{1+e} \gamma_{w}-\frac{w}{1+w} \gamma\right)
$$

where $\gamma_{a}$ is the average unit weight of soil between the water levels before and after drawdown, $\gamma_{w}$ is the unit weight of water, $e$ is the void ratio, $w$ is water content of the soil, and $r_{u}$ is the pore water pressure coefficient.

When the pore water pressure has not yet dissipated, $\gamma_{a}$ equals saturated unit weight. When the pore water pressure has completely dissipated, $\gamma_{a}$ equals the unsaturated unit weight, which means that the water level in the sliding mass is consistent with the reservoir.

The factor of safety can be obtained by superimposing three independent slope models with the same slope angle $\beta$. Model 1 is the simple homogenous slope $A_{1} C_{1}$ with unsaturated unit weight as the original Taylor's model shown in Figure 1. Model 2 is the slope $\mathrm{AB}_{2} \mathrm{C}_{2}$, where $\mathrm{H}_{2}$ is the slope height, $2 \alpha_{2}$ is the central angle of the failure surface $\operatorname{arc} \mathrm{AB}_{2}$, and $\lambda_{2}$ is the inclination angle of the chord $\mathrm{AB}_{2}$. The unit weight of $\mathrm{AB}_{2} \mathrm{C}_{2}$ equals the difference between $\gamma_{a}$ and $\gamma_{1}$, as shown in Figure 2. Model 3 is the slope $\mathrm{AB}_{3} \mathrm{C}_{3}$, where $H_{3}$ is the slope height, $2 \alpha_{3}$ is the central angle of the failure surface $\operatorname{arc} A_{3}$, and $\lambda_{3}$ is the inclination angle of the chord $A_{3}$. The unit weight of $\mathrm{AB}_{3} \mathrm{C}_{3}$ equals the difference of $\gamma^{\prime}$ (the effective unit weight) and $\gamma_{a}$, as shown in Figure 3 . It is necessary to take the pore water pressure between the water levels before and after drawdown into consideration in model 3. Because the pore water pressure passes through the center of the $\varphi$-circle, its moment is zero. Superimposing the gravity and gravity moment of the three models, we can obtain the practical gravity $\mathrm{W}$ and its moment $W_{d}$. Based on the derivation of Taylor, the analytical expression for the factor of safety in water drawdown slopes can be established.

Deduction of the gravity and gravity moment for the latter two models is listed in Appendix A. The polygon of force equilibrium in the case of water declining is shown in Figure 4, in which there is an added pore water pressure $P_{w}$ on the failure surface compared with Taylor's original model. There are four forces acting on the sliding mass, the weight $W$, the resultant cohesion $C$, the resultant normal force $P^{\prime}$, and the pore water pressure $P_{w}$. The magnitude and direction of force $C$ are the same as Taylor's original model. The magnitude of $W$ is obtained by equation (A.11) in Appendix A. $P^{\prime}$ is tangent to the $\varphi$-circle and has an angle $v$ with $W$. The expression for angle $v$ is the same as Taylor's model. $P_{w}$ passes through the center of $\varphi$-circle. The resultant of $P^{\prime}$ and $P_{w}$ is assumed to be $P$. The angle $\mu$ can be calculated using equation (14). The detailed deduction of equation (14) and the related terms in equation (14) is listed in Appendix B.

$$
\mu=\arcsin \frac{P_{w} \cos \left(\lambda_{1}-\delta-v\right)}{\sqrt{P^{, 2}+P_{1}^{2}-2 P^{\prime} \times P_{w} \sin \left(\lambda_{1}-\delta-v\right)}}
$$




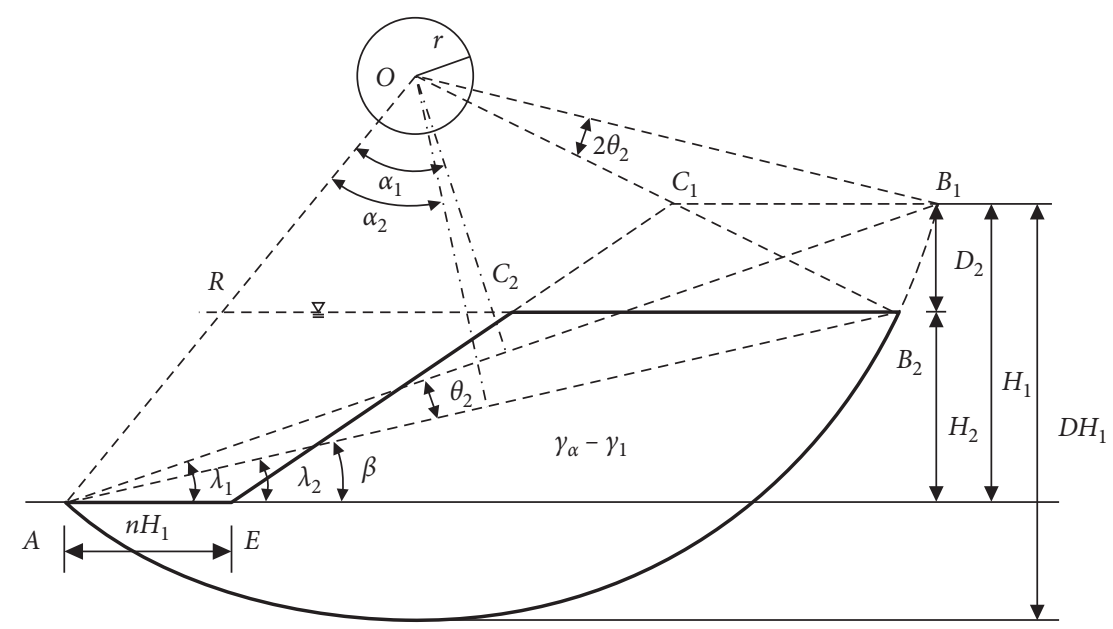

FIgURe 2: A slope model showing water level and the geometric denomination.

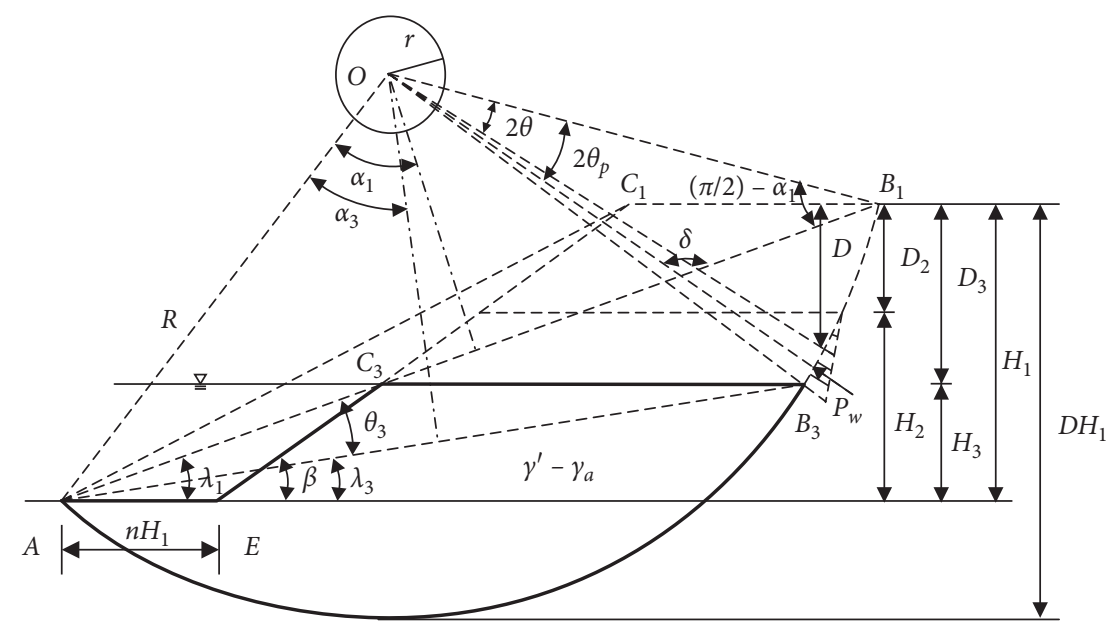

Figure 3: A slope model showing water drawdown and the geometric denomination.

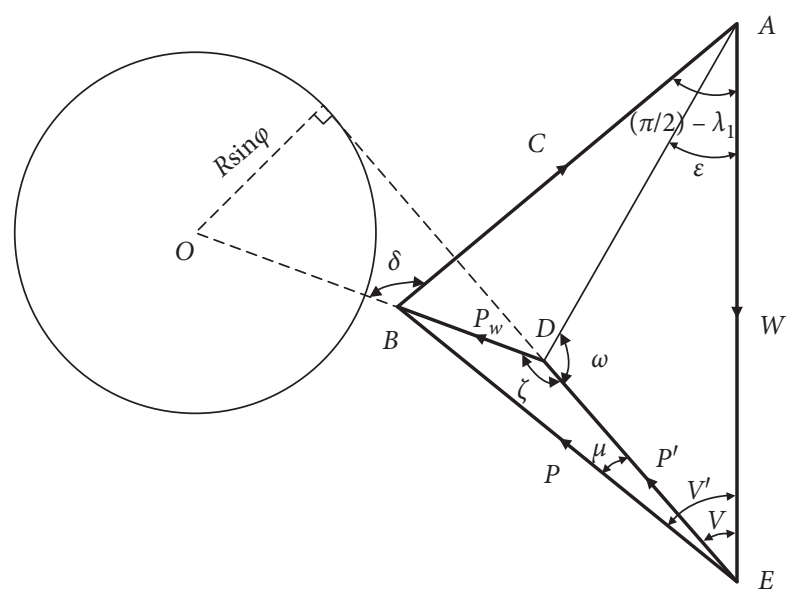

Figure 4: Polygon of force balance in water drawdown slopes. 
The general expression for the factor of safety in the case of water drawdown is shown in equation (15). The detailed reduction is listed in Appendix C.

$$
F=\left(\frac{c}{H_{1}}\right) \frac{2 \cot \lambda_{1} \cot \nu^{\prime}+2}{\sum_{i=1}^{3} \gamma_{i}\left[K_{i}^{2} f\left(\lambda_{i}, \alpha_{i}\right)-2 K_{i} n\right]}, \quad i=1,2,3
$$

where

$$
v^{\prime}=v+\mu
$$

Based on the general form, the factor of safety for six cases can be simplified as below.

For a simple homogenous slope as Taylor's model, under the conditions of $H_{2}=H_{3}=0, K_{2}=K_{3}=0, P_{w}=0$, and $v^{\prime}=v$, equation (15) can be simplified as

$$
F=\left(\frac{c}{H_{1}}\right) \frac{2 \cot \lambda_{1} \cot \nu+2}{\gamma_{1}\left[f\left(\lambda_{1}, \alpha_{1}\right)-2 n\right]}
$$

For the completely submerged slope, under the conditions of $H_{2}=H_{3}=H_{1}, K_{2}=K_{3}=1, r_{u}=0, \gamma_{2}=0, \gamma_{3}=\gamma^{\prime}-\gamma_{1}$,
$P_{w}=0$, and $v^{\prime}=v$, equation (15) can be simplified as the same as equation (17) in which $\gamma_{1}$ is replaced with $\gamma^{\prime}$.

For the partially submerged slope, under the conditions of $H_{2}=H_{3}, K_{2}=K_{3}, r_{u}=0, \gamma_{2}=0, \gamma_{3}=\gamma^{\prime}-\gamma_{1}, P_{w}=0$, and $v^{\prime}=v$, equation (15) can be simplified as

$$
F=\left(\frac{c}{H_{1}}\right) \frac{2 \cot \lambda_{1} \cot \nu+2}{\gamma_{1}\left[f\left(\lambda_{1}, \alpha_{1}\right)-2 n\right]+\left(\gamma^{\prime}-\gamma_{1}\right)\left[K_{3}^{2} f\left(\lambda_{2}, \alpha_{2}\right)-2 K_{3} n\right]} \text {. }
$$

For the slope where water level declines very slowly or the soil is highly permeable, the water level in the slope is synchronous with the reservoir water level. In this case, the expression for the factor of safety is the same as equation (18).

For the slope that experiences water drawdown with a decrease of water level, the soil in the zone between the water levels before and after the water drawdown is regarded to be saturated due to capillarity. In this case, $\gamma_{2}=\gamma_{\text {sat }}-\gamma_{1}$, $\gamma^{3}=-\gamma_{w}, P_{w}=0$, and $v^{\prime}=v$, and equation (15) can be simplified as

$$
F=\left(\frac{c}{H_{1}}\right) \frac{2 \cot \lambda_{1} \cot \nu+2}{\gamma_{1}\left[f\left(\lambda_{1}, \alpha_{1}\right)-2 n\right]+\left(\gamma_{\mathrm{sat}}-\gamma_{1}\right)\left[K_{2}^{2} f\left(\lambda_{2}, \alpha_{2}\right)-2 K_{2} n\right]-\gamma_{w}\left[K_{3}^{2} f\left(\lambda_{3} \alpha_{3}\right)-2 K_{3} n\right]}
$$

For the slope having water sudden drawdown, the soil between the water levels before and after drawdown is saturated and the pore water pressure on the failure arc has not dissipated. In this case, $r_{u}=\gamma_{w} / \gamma, \gamma_{2}=\gamma_{\text {sat }}-\gamma_{1}$, and $\gamma_{3}=-\gamma_{w}$, and equation (15) can be simplified as

$$
F=\frac{c}{H_{1}} \frac{2 \cot \lambda_{1} \cot \nu^{\prime}+2}{\gamma_{1}\left[f\left(\lambda_{1}, \alpha_{1}\right)-2 n\right]+\left(\gamma_{\text {sat }}-\gamma_{1}\right)\left[K_{2}^{2} f\left(\lambda_{2}, \alpha_{2}\right)-2 K_{2} n\right]-\gamma_{w}\left[K_{3}^{2} f\left(\lambda_{3}, \alpha_{3}\right)-2 K_{3} n\right]}
$$

\section{Charts for the Factor of Safety}

The failure surface arc has three degrees of freedom, namely, $n, \alpha_{1}$, and $\lambda_{1}$. Given reasonable domains for the three degrees of freedom, the minimum factor of safety corresponding to the critical failure surface can be searched with any optimum method such as the golden-section method. In this study, the failure surface is determined using the computer program accomplished by the authors.

Considering the simple homogenous slope first, the factor of safety has a linear relationship with $c / \gamma \mathrm{H}_{1}$ as shown in Equation (15). Given $c / \gamma H_{1}=0.01$ and 0.10 , respectively, the minimum factors of safety against a range of slope inclinations and internal friction angles are shown as charts in Figure 5.

An example is described to explain how to use the charts. The slope height $\mathrm{H} 1$ is $50 \mathrm{~m}$; the slope inclination angle $\beta$ is $30^{\circ}$; the cohesion $c$ is $30 \mathrm{kPa}$; the internal friction $\varphi$ is $25^{\circ}$; and the unit weight $\gamma$ is $17 \mathrm{kN} / \mathrm{m}^{3}$. With the given $\beta$ and $\varphi$ values, $F$ is 1.04 when $c / \gamma H_{1}=0.01$ and is 1.89 when $c / \gamma H_{1}=0.10$ based on Figure 5(a) and Figure 5(b). Afterward, $c / \gamma H_{1}$ is 0.034 based on the given $c, \gamma$, and $H_{1}$ values. $F$ of the slope is 1.27 by interpolation between 1.04 and 1.89 .

In Taylor's chart, the factor of safety is defined as the ratio of actual cohesion to critical cohesion. To find the reliable factor of safety, it needs to adjust $F$ for getting equal values to fit both the friction angle and the stability number, which is a tedious process in using the chart. Figure 5 provides a direct and simple way to determine the factor of safety.

For a submerged slope with the static water level, $F$ has a linear relationship with $c / H_{1}$ as shown in equation (19). Given $c / H_{1}=1.0$ and $2.0, \gamma=17.5 \mathrm{kN} / \mathrm{m}^{3}, e=0.67, w=28 \%$, $\gamma_{w}=9.81 \mathrm{kN} / \mathrm{m}^{3}$, and $K_{2}$ is a series of values between $0-1.0$, the minimum $F$ with a range of $\beta$ and $\varphi$ is calculated. The results are presented as charts in Figure 6 . It suggests that for the steep slope (i.e., $\beta \geq 60^{\circ}$ ), the factor of safety increases monotonously with the rise of water level; for the gentle slopes, the factor of safety decreases at the beginning and then increases with the rise of water level. 


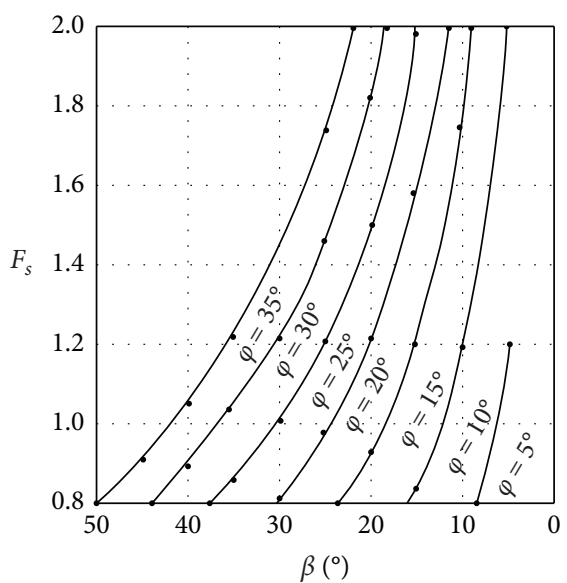

(a)

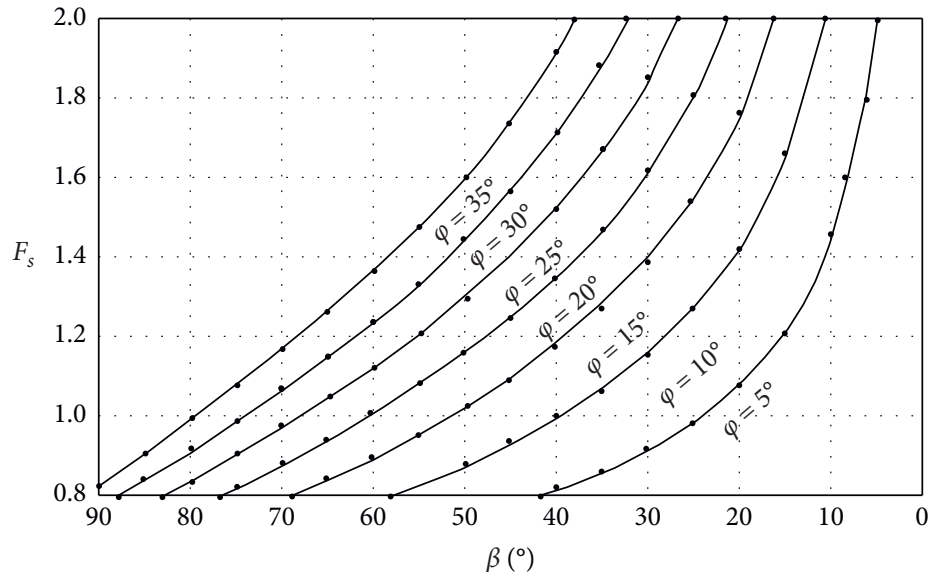

(b)

Figure 5: Factor of safety charts for homogenous slopes. (a) $c / \gamma H_{1}=0.01$. (b) $c / \gamma H_{1}=0.1$.

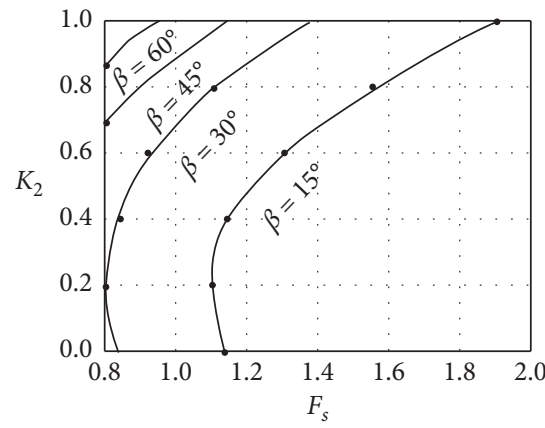

(a)

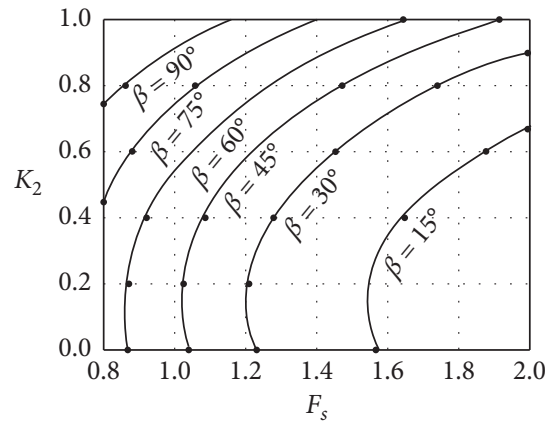

(d)

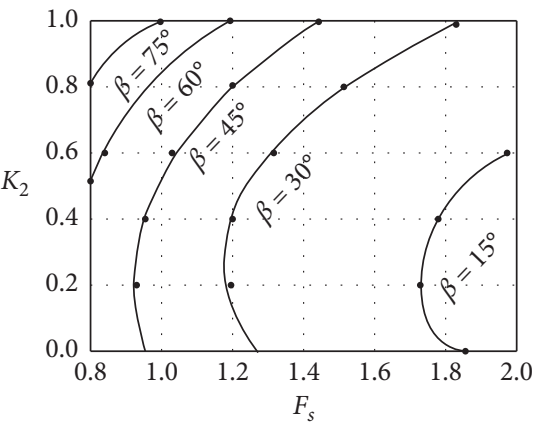

(b)

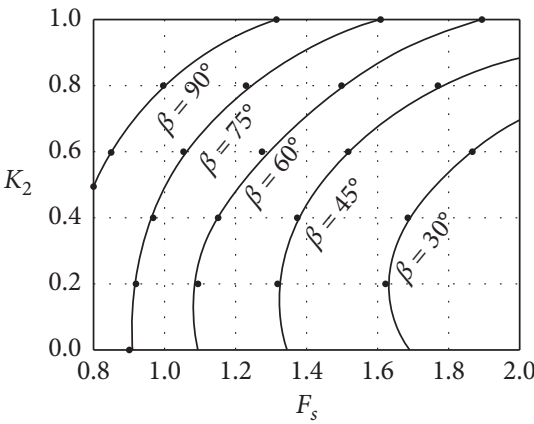

(e)

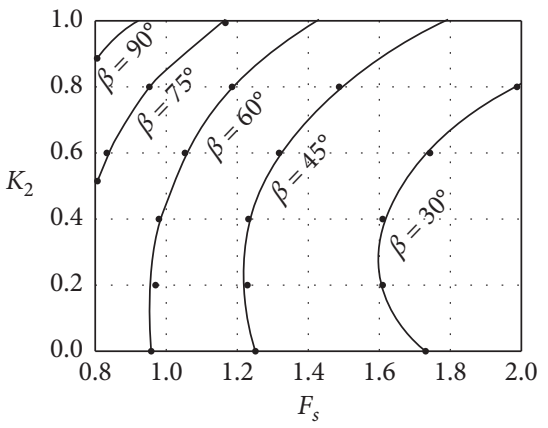

(c)

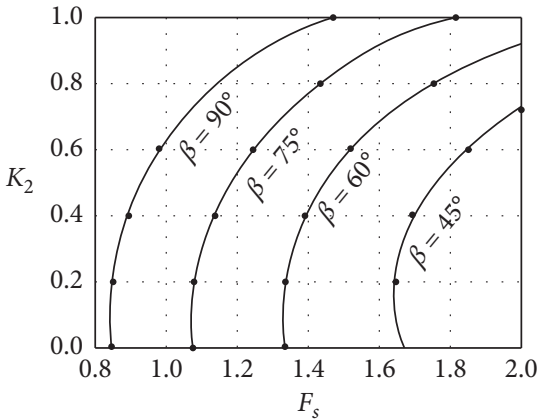

(f)

Figure 6: Factor of safety charts for different static water levels: (a) $c / H_{1}=1.0, \varphi=10^{\circ}$; (b) $c / H_{1}=1.0, \varphi=20^{\circ}$; (c) $c / H_{1}=1.0, \varphi=30^{\circ}$; (d) $c / H_{1}=2.0, \varphi=10^{\circ}$; (e) $c / H_{1}=2.0, \varphi=20^{\circ}$; (f) $c / H_{1}=2.0, \varphi=30^{\circ}$.

For the case of water sudden drawdown, assume $H_{1}=50 \mathrm{~m}, \gamma=16 \mathrm{kN} / \mathrm{m}^{3}, e=0.43, w=28 \%, \gamma_{w}=9.81 \mathrm{kN} / \mathrm{m}^{3}$, $r_{u}=0, w=15 \%, \beta=30^{\circ}, \varphi=20^{\circ}$, and a range of original water levels $\mathrm{H}_{2}$ and the terminal water levels $\mathrm{H}_{3}$ between $10 \mathrm{~m}$ and $45 \mathrm{~m}$. Calculations are carried out with the orthogonal values of $K_{2}$ and $K_{3}$. The results are presented in Figure 7. The top curve shows the factor of safety against static water where $K_{2}=K_{3}$. The curves that intersect with the top curve show the factor of safety against the water drawdown from $K_{2}$ to $K_{3}$. It suggests in this example that the factor of safety increases with the rise of the static water level. However, it decreases with the water sudden drawdown for all the original water levels. The larger the water level drawdown is, the lower the factor of safety is. The results are in agreement with the results by Cojean and Fleurisson [20] and Cojean and Caï [21].

The results in Figures 6 and 7 are calculated under given parameters. It is difficult to make general charts for the factor of safety for all slopes. However, it is easy and practical to figure out using the computer program. 


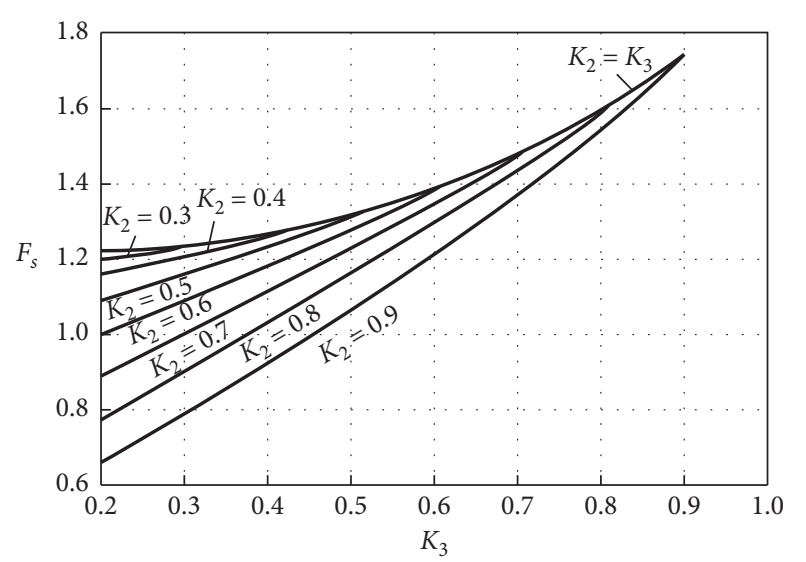

FIgURE 7: Factor of safety charts for water sudden drawdown case.

\section{Conclusions}

Based on Taylor's $\varphi$-circle method, a general expression for the factor of safety of the slope in the water drawdown case is proposed. Some typical cases can be simplified with the general expression, such as homogenous, partly submerged, and completely submerged slopes, as well as water sudden drawdown and slow drawdown slopes. A computer program written by the authors is used to search the failure surface and figure out the minimum factor of safety.

For simple homogenous slopes, the charts are designed for determining the factor of safety with aid of interpolation between two values of $c / \gamma H_{1}$ that has a linear relation with $F$. It avoids the tedious process of iteration with Taylor's stability number chart.

For the slopes with static water level or water drawdown, the factor of safety is a linear function with $c / H_{1}$. It is difficult to give a set of general charts due to the additional free variables such as unit weights above and below the water level. However, it is easy to obtain using the proposed computer programs. Some typical cases have been calculated and shown as charts in the paper.

\section{Appendix}

\section{A. Gravity and Gravity Moment Calculation for the Slope of Water Drawdown}

For the simple slope model shown in Figure 1, the gravity and gravity moment of the sliding mass have been derived by Taylor [1] and given in the text as equations (4) and (11).

For the model shown in Figure 2, the unit weight of the sliding mass is

$$
\gamma_{2}=\gamma_{a}-\gamma_{1}
$$

Let

$$
\theta_{2}=\lambda_{1}-\lambda_{2}
$$

From Figure 1, it can be seen that

$$
\begin{aligned}
\theta_{2} & =\alpha_{1}-\alpha_{2} . \\
D_{2} & =2 R \sin \theta_{2} \sin \left(\lambda_{1}+\alpha_{1}-\theta_{2}\right) .
\end{aligned}
$$

Then,

$$
\begin{aligned}
& \theta_{2}=\frac{\lambda_{1}+\alpha_{1}-\arccos \left[\left(D_{2} / R\right)+\cos \left(\lambda_{1}+\alpha_{1}\right)\right]}{2} . \\
& \lambda_{2}=\frac{\lambda_{1}-\alpha_{1}+\arccos \left[\left(D_{2} / R\right)+\cos \left(\lambda_{1}+\alpha_{1}\right)\right]}{2} . \\
& \alpha_{2}=\frac{\alpha_{1}-\lambda_{1}+\arccos \left[\left(D_{2} / R\right)+\cos \left(\lambda_{1}+\alpha_{1}\right)\right]}{2} .
\end{aligned}
$$

For the model shown in Figure 3, the unit weight is

$$
\gamma_{3}=\gamma^{\prime}-\gamma_{\mathrm{a}}
$$

Similarly, we have

$$
\begin{array}{ll}
\theta_{i}=\frac{\lambda_{1}+\alpha_{1}-\arccos \left[\left(D_{i} / R\right)+\cos \left(\lambda_{1}+\alpha_{1}\right)\right]}{2}, & i=1,2,3, \\
\lambda_{i}=\frac{\lambda_{1}-\alpha_{1}+\arccos \left[\left(D_{i} / R\right)+\cos \left(\lambda_{1}+\alpha_{1}\right)\right]}{2}, & i=1,2,3, \\
\alpha_{i}=\frac{\alpha_{1}-\lambda_{1}+\arccos \left[\left(D_{i} / R\right)+\cos \left(\lambda_{1}+\alpha_{1}\right)\right]}{2}, & i=1,2,3,
\end{array}
$$

$f\left(\lambda_{i}, \alpha_{i}\right)=\left(\frac{1}{2}\right) \csc ^{2} \lambda_{i}\left(\alpha_{i} \csc ^{2} \alpha_{i}-\cot \alpha_{i}\right)+\cot \lambda_{i}-\cot \beta$.

Let

$$
K_{i}=\left(\frac{H_{1}-D_{i}}{H_{1}}\right)=\left(\frac{H_{i}}{H_{1}}\right), \quad i=1,2,3 .
$$

Similar to equations (4) and (11) in the text, we have

$$
W_{i}=K_{i}^{2}\left(\frac{\gamma_{i} H_{1}^{2}}{2}\right) f\left(\lambda_{i}, \alpha_{i}\right)-K_{i} \gamma_{i} n H_{1}^{2}, \quad i=1,2,3,
$$

$W_{i} d_{i}=K_{i}^{3}\left(\frac{\gamma_{i} H_{1}^{3}}{4}\right) g\left(\lambda_{i}, \alpha_{i}\right)-K_{i}\left(\frac{\gamma_{i} n H_{1}^{3}}{2}\right)\left[K_{i} \psi\left(\lambda_{i}, \alpha_{i}\right)+n\right]$,

$$
i=1,2,3 \text {. }
$$

Referring to equations (9) and (10) in the text, we have

$$
\begin{aligned}
\psi\left(\lambda_{i}, \alpha_{i}\right)= & \cot \beta+\cot \alpha_{i}-\cot \lambda_{i}, \quad i=1,2,3 \\
g\left(\lambda_{i}, \alpha_{i}\right)= & \left(\frac{1}{3}\right)\left(1-2 \cot ^{2} \beta\right)+\cot \beta\left(\cot \lambda_{i}-\cot \alpha_{i}\right) \\
& +\cot \lambda_{i} \cot \alpha_{i}, \quad i=1,2,3 .
\end{aligned}
$$

Superimposing the gravity and gravity moment of the three models, we can obtain the practical gravity and gravity moment. 


$$
\begin{gathered}
W=\sum_{i=1}^{3} K_{i}^{2}\left(\frac{\gamma_{i} H_{1}^{2}}{2}\right) f\left(\lambda_{i}, \alpha_{i}\right)-K_{i} \gamma_{i} n H_{1}^{2}, \quad i=1,2,3, \\
\begin{array}{cl}
W d= & \sum_{i=1}^{3} K_{i}^{3}\left(\frac{\gamma_{i} H_{1}^{3}}{4}\right) g\left(\lambda_{i}, \alpha_{i}\right)-K_{i}\left(\frac{\gamma_{i} n H_{1}^{3}}{2}\right) \\
\cdot\left[K_{i} \psi\left(\lambda_{i}, \alpha_{i}\right)+n\right], \quad i=1,2,3 .
\end{array}
\end{gathered}
$$

Substituting equation (A.11) into equation (A.12), we get $\frac{H_{1}}{2 d}=\frac{\sum_{i=1}^{3} \gamma_{i}\left[K_{i}^{2} f\left(\lambda_{i}, \alpha_{i}\right)-2 K_{i} n\right]}{\sum_{i=1}^{3} \gamma_{i}\left\{K_{i}^{3} g\left(\lambda_{i}, \alpha_{i}\right)-2 K_{i} n\left[K_{i} \psi\left(\lambda_{i}, \alpha_{i}\right)+n\right]\right\}}, \quad i=1,2,3$.

\section{B. Deduction of the Angle $\mu$ between $P^{\prime}$ and $P$}

Based on equation (12) in the text, the resultant pore water pressure $P_{w}$ on the failure surface between water levels before and after drawdown can be estimated by

$$
P_{w}=\int_{l_{2}}^{l_{3}} r_{u} \gamma\left(D-D_{2}\right) \cos \left(2 \theta-2 \theta_{P}\right) \mathrm{d} l,
$$

where $l_{2}\left(l_{3}\right)$ is the arc length of the failure surface from point $B_{1}$ to the corresponding water depth $D_{2}\left(D_{3}\right), 2 \theta$ is the center angle corresponding to the depth $D$, and $2 \theta_{p}$ is the center angle corresponding to the point of the resultant force $P_{w}$.

From Figure 3, it results that

$$
\begin{aligned}
& D=R\left[\cos \left(\lambda_{1}+\alpha_{1}-2 \theta\right)-\cos \left(\lambda_{1}+\alpha_{1}\right)\right] . \\
& \mathrm{d} l=\operatorname{Rd} 2 \theta .
\end{aligned}
$$

D2 and D3 can be calculated with equation (B.1) as $\theta$ is given the value as $\theta_{2}$ or $\theta_{3}$.

The acting position of $P_{w}$ is approximately on $\left(\mathrm{H}_{2}-\mathrm{H}_{3}\right) / 3$ above the water level after drawdown or $\left(2 D_{3}-D_{2}\right) / 3$ below the top ground of the slope as shown in Figure 3. $\left(2 D_{3}-D_{2}\right) / 3$ can be expressed as

$$
\left(\frac{2 D_{3}-D_{2}}{3}\right)=R\left[\cos \left(\lambda_{1}+\alpha_{1}-2 \theta_{P}\right)-\cos \left(\lambda_{1}+\alpha_{1}\right)\right] .
$$

So,

$$
\theta_{P}=\frac{\lambda_{1}+\alpha_{1}-\arccos \left[\left(2 D_{3}-D_{2} / 3 R\right)+\cos \left(\lambda_{1}+\alpha_{1}\right)\right]}{2} .
$$

Substituting equations (B.2), (B.3), and (B.5) into equation (B.1), $P_{w}$ can be deduced by integration and the result is

$$
\begin{aligned}
P_{w}= & \frac{1}{4} r_{u} \gamma R^{2}\left[\sin \left(\lambda_{1}+\alpha_{1}+2 \theta_{p}\right)\left(\cos 4 \theta_{2}-\cos 4 \theta_{3}\right)\right. \\
& \left.+\cos \left(\lambda_{1}+\alpha_{1}+2 \theta_{p}\right)\left(\sin 4 \theta_{3}-\sin 4 \theta_{2}+4 \theta_{3}-4 \theta_{2}\right)\right] \\
& -r_{u} \gamma R\left[R \cos \left(\lambda_{1}+\alpha_{1}\right)+D_{2}\right]\left[\sin 2\left(\theta_{3}-\theta_{p}\right)\right. \\
& \left.-\sin 2\left(\theta_{2}-\theta_{p}\right)\right] .
\end{aligned}
$$

Referring to Figure 3, there is

$$
\delta+\left(\frac{\pi}{2}\right)-\left(\alpha_{1}+2 \theta_{p}\right)=\pi .
$$

So, the angle $\delta$ between $P_{w}$ and $C$ is

$\delta=\left(\frac{\pi}{2}\right)-\left(\lambda_{1}+\arccos \right)\left[\left(\frac{2 D_{1}-D_{2}}{3 R}\right)+\cos \left(\lambda_{1}+\alpha_{1}\right)\right]$.

By the cosine law in triangle ABD in Figure 4,

$$
\mathrm{AD}=\sqrt{C^{2}+P_{w}^{2}-2 C \times P_{w} \cos \delta} .
$$

By the sinusoidal law in triangle ADE in Figure 4,

$$
\left(\frac{\sin v}{\mathrm{AD}}\right)=\left(\frac{\sin \omega}{W}\right)=\left(\frac{\sin (\pi-\omega-v)}{P^{\prime}}\right) .
$$

Substituting equation (B 9) into equation (B.10), we get

$$
\begin{gathered}
\omega=\arcsin \frac{W \sin v}{\sqrt{C^{2}+P_{w}^{2}-2 C \times P_{w} \cos \delta}}, \\
P^{\prime}=\frac{\sin (\omega+v) \sqrt{C^{2}+P_{w}^{2}-2 C \times P_{w} \cos \delta}}{\sin v} .
\end{gathered}
$$

By the cosine law and sinusoidal law in triangle BDE in Figure 4, there are

$$
\begin{aligned}
P & =\sqrt{P^{\prime 2}+P_{w}^{2}-2 P^{\prime} \times P_{w} \cos \zeta} \\
\left(\frac{\sin \mu}{P_{w}}\right) & =\left(\frac{\sin \zeta}{P}\right), \\
\zeta & =\left(\frac{\pi}{2}\right)-\left(\lambda_{1}+\delta+v\right) .
\end{aligned}
$$

So, equation (B 13) becomes

$$
P=\sqrt{P^{\prime 2}+P_{w}^{2}-2 P^{\prime} \times P_{w} \sin \left(\lambda_{1}-\delta-v\right) .}
$$

Substituting equations (B 13) and (B 16) into equation (B $14)$, we get 


$$
\mu=\arcsin \frac{P_{w} \cos \left(\lambda_{1}-\delta-v\right)}{\sqrt{P^{\prime 2}+P_{w}^{2}-2 P^{\prime} \times P_{w} \sin \left(\lambda_{1}-\delta-v\right)}} .
$$

\section{Deduction of the Factor of Safety}

A common definition of the factor of safety is the ratio of the shear strength of the soil to the shear strength mobilized to retain the limit equilibrium:

$$
F=\left(\frac{c}{c_{d}}\right)=\left(\frac{\tan \varphi}{\tan \varphi_{d}}\right)
$$

in which $F$ is the factor of safety, $c$ is the cohesion, $c_{d}$ is the mobilized soil cohesion, $\varphi$ is the internal friction angle, and $\varphi_{d}$ is the mobilized internal friction angle.

The mobilized internal angle is

$$
\varphi_{d}=\arctan \left(\frac{\tan \varphi}{F}\right) \text {. }
$$

Referring to Taylor [1], we have

$$
v=u-\arcsin \left(\left(\frac{H}{2 d}\right) \sin u \csc \lambda_{1} \csc \alpha_{1} \sin \varphi_{d}\right),
$$

where

$$
\cot u=\left(\frac{H}{2 d}\right) \alpha_{1} \sec \lambda_{1} \csc \lambda_{1} \csc ^{2} \alpha_{1}-\left(\tan \lambda_{1}\right)
$$

Using sinusoidal law and referring to Figure 4, there is

$$
\frac{W}{\sin \left((\pi / 2)+\lambda_{1}-v^{\prime}\right)}=\left(\frac{C}{\sin v^{\prime}}\right)
$$

where

$$
\begin{aligned}
& v^{\prime}=v+\mu, \\
& C=\left(\frac{2}{F}\right) c R \sin \alpha_{1},
\end{aligned}
$$

In which

$$
R=\left(\frac{H_{1}}{2}\right) \csc \alpha_{1} \lambda_{1}
$$

Substituting equation ( $\mathrm{C}$ 4) into ( $\mathrm{C} 3$ ) and then substituting equations (C 3) and (A 11) into (C 1), the factor of safety can be deduced as

$$
F=\left(\frac{c}{H_{1}}\right) \frac{2 \cot \lambda_{1} \cot \nu^{\prime}+2}{\sum_{i=1}^{3} \gamma_{i}\left[K_{i}^{2} f\left(\lambda_{i}, \alpha_{i}\right)-2 K_{i} n\right]}, \quad i=1,2,3 .
$$

The angle $\mu$ in equation (C 2) is deduced in Appendix B.

\section{Data Availability}

The data used to support the findings of this study are included in the article.

\section{Conflicts of Interest}

The authors declare that they have no conflicts of interest.

\section{Acknowledgments}

The authors acknowledge the funding received from the National Natural Science Foundation of China (program nos. 41877242 and 41772278 ) and the National Key R\&D Program of China (2017YFC1501302) which supported this study.

\section{References}

[1] D. W. Taylor, "Stability of earth slopes," Journal of the Boston Society of Civil Engineers, vol. 24, no. 3, pp. 197-246, 1937.

[2] D. V. Griffiths, J. Huang, and G. A. Fenton, "Influence of spatial variability on slope reliability using 2-D random fields," Journal of Geotechnical and Geoenvironmental Engineering, vol. 135, no. 10, pp. 1367-1378, 2009.

[3] H. Zhu, D. V. Griffiths, G. A. Fenton, and L. M. Zhang, "Undrained failure mechanisms of slopes in random soil," Engineering Geology, vol. 191, pp. 31-35, 2015.

[4] R. E. Gibson and N. Morgenstern, "A note on the stability of cuttings in normally consolidated clays," Géotechnique, vol. 12, no. 3, pp. 212-216, 1962.

[5] J. H. Hunter and R. L. Schuster, "Stability of simple cuttings in normally consolidated clays," Géotechnique, vol. 18, no. 3, pp. 372-378, 1968.

[6] D. V. Griffiths and X. Yu, "Another look at the stability of slopes with linearly increasing undrained strength," Géotechnique, vol. 65, no. 10, pp. 824-830, 2015.

[7] P. Li, Q. Zhang, X. Hou et al., "Stability analysis of an undrained soil slope against the linearly increasing shear strength," Hydrogeology \& Engineering Geology, vol. 43, no. 3, pp. 59-65, 2016, in Chinese.

[8] R. Baker, R. Shukha, V. Operstein, and S. Frydman, "Stability charts for pseudo-static slope stability analysis," Soil Dynamics and Earthquake Engineering, vol. 26, no. 9, pp. 813823, 2006.

[9] P. P. Sahoo and S. K. Shukla, “Taylor's slope stability chart for combined effects of horizontal and vertical seismic coefficients," Géotechnique, vol. 69, no. 4, pp. 344-354, 2019.

[10] C. Sun, J. Chai, B. Ma, T. Luo, Y. Gao, and H. Qiu, "Stability charts for pseudostatic stability analysis of 3D homogeneous soil slopes using strength reduction finite element method," Advances in Civil Engineering, vol. 2019, Article ID 6025698, 18 pages, 2019.

[11] W. Liu, T. Li, and P. Li, "Numerical analysis for failure mode and ultimate bearing capacity of striping footings on the crest of undrained slopes," Journal of Engineering Geology, vol. 24, no. 2, pp. 197-203, 2016, (in Chinese).

[12] R. Baker and Y. Tanaka, "A convenient alternative representation of Taylor's stability chart," Slope Stability Engineering, vol. 1, pp. 253-257, 1999.

[13] R. Baker, "A second look at taylor's stability chart," Journal of Geotechnical and Geoenvironmental Engineering, vol. 129, no. 12, pp. 1102-1108, 2003. 
[14] T. Steward, N. Sivakugan, S. K. Shukla, and B. M. Das, "Taylor's slope stability charts revisited," International Journal of Geomechanics, vol. 11, no. 4, pp. 348-352, 2011.

[15] D. V. Griffiths and C. M. Martin, "Critical failure mechanisms in relatively flat undrained slopes," Géotechnique Letters, pp. 1-5, 2019.

[16] R. L. Michalowski and T. Martel, "Stability charts for 3D failures of steep slopes subjected to seismic excitation," Journal of Geotechnical and Geoenvironmental Engineering, vol. 137, no. 2, pp. 183-189, 2011.

[17] K. Lim, A. V. Lyamin, M. J. Cassidy et al., "Three-dimensional slope stability charts for frictional fill materials placed on purely cohesive clay," International Journal of Geomechanics, vol. 16, no. 2, Article ID 04015042, 2016.

[18] C. Sun, J. Chai, Z. Xu et al., "3D stability charts for convex and concave slopes in plan view with homogeneous soil based on the strength-reduction method," International Journal of Geomechanics, vol. 17, no. 5, Article ID 06016034, 2017.

[19] R. L. Michalowski, "Stability charts for uniform slopes," Journal of Geotechnical and Geoenvironmental Engineering, vol. 128, no. 4, pp. 351-355, 2002.

[20] R. Cojean and J. A. Fleurisson, “"Influence de la structure géologique sur la stabilité de versants progressivement submergés par la montée d'un plan d'eau," in Proceedings of the 6th International IAEG Congress, pp. 2113-2132, Amsterdam, Netherlands, August 1990.

[21] R. Cojean and Y. J. Caï, "Analysis and modeling of slope stability in the Three-Gorges Dam reservoir (China) - the case of Huangtupo landslide," Journal of Mountain Science, vol. 8, no. 2, pp. 166-175, 2011.

[22] T. Li, C. Zhang, P. Xu et al., "Stability ssessment and tabilizing pproaches for the Majiagou Landslide, ndergoing the ffects of ater evel luctuation in the Three Gorges Reservoir Area," Landslide Disaster Mitigation in the Three Gorges Reservoir, China, pp. 2113-2132, Springer, Berlin, Germany, 2009. 